\title{
Pharmaceutical Liaison
}

National Cancer Institute

\section{Source}

National Cancer Institute. Pharmaceutical Liaison. NCI Thesaurus. Code C51839.

A person who initiates and maintains a contact or connection pertaining to pharmacy. 